\title{
Optical Network Traffic Control Algorithm under Variable Loop Delay: A Simulation Approach
}

\author{
Manoj Kr DUTTA, Vinod Kumar CHAUBEY \\ Electrical and Electronics Engineering Department, Birla Institute of Technology \& Science, \\ Pilani, Rajasthan, India \\ Email:mkdutta13@gmail.com,vkc@bits.pilani.ac.in \\ Received May 9, 2009; revised July 16, 2009; accepted August 22, 2009
}

\begin{abstract}
In this paper we present a concept of new architectural model consisting of multiple loop delay to increase the throughput. The simulated behavior of an optical node has been realized by using an $\mathrm{n} \mathrm{m}$ optical switch and recirculating optical delay lines. This investigation infers the scaling behaviors of the proposed architecture to maintain efficient use of the buffer under Poisson traffic loading. The analysis also reports the traffic handling capacity for the given complexity of the node architectural design.
\end{abstract}

Keywords: Fiber Delay Line, Recirculation, Traffic, Throughput

\section{Introduction}

ALL-OPTICAL communication has been proposed as a promising candidate for providing high-speed networking [1-4] owing to huge bandwidth of optical channels. Wide bandwidth available in low attenuation window in the optical fiber can be divided into a number of independent wavelength channels as per network standard and specification leading to SONET, SDH or wavelength division-multiplexing (WDM) based all optical network system [5-7]. Evidently to support such all optical control in these networks several technologies have been proposed for efficient networking viz., broadcast and select, wavelength routing, optical packet switching (OPS), and optical burst switching [8-10]. In an OPS network, optical interconnect (or optical switch) forwards the packets to their destinations involving programmable switch fabric and control circuitry and thereby support in packet contention resolution. However in a WDM interconnect, output contention arises when more than one packet on the same wavelength are destined to the same output fiber at the same time. To resolve this contention one will have to either temporarily store some of the packets in a buffer, or to convert wavelengths to some available wavelengths by wavelength converters [11-13]. Obviously optical buffers, wavelength converters add the complexity by enhancing the installation and recurring cost of the system. However allocating some dedicated buffers for each output fiber which can share a common optical delay line (ODL) buffer pool [5-6] will essentially reduce the cost and complexity as well. In a WDM a packet that cannot be directly sent to the output fiber is sent back to one of the delay lines for recirculation and after being delayed by some specific time, that packet will come out of the delay line to compete for throughput with the newly arriving packets. In case of unsuccessful throughput it gets back into the delay line for the next round trip with additional delays.

In the proposed model a node has been considered with more input channels than output channels and the maximum capacity of this node is decided by the available output channel. It is assumed that arriving packets are destined to their respective destinations based on First Come First Serve (FCFS) scheduling policy. In this way we can avoid the continuous recirculation of some packet in the delay line. Packets that arrive in the meantime are also sent to delay line. The node includes finite capacity buffer and multiple delay lines arranged in synchronized mode.

\section{Node Architecture Design and Modeling}

The packet switching has its own (unique) issues in optical networks. In an optical packet-switched network, contention occurs due to unavailability of free output wavelength. In electrical packet-switched networks, contention is resolved with the store-and-forward technique, which requires the packets losing the contention to be stored in a memory bank and to be sent out at a later time 


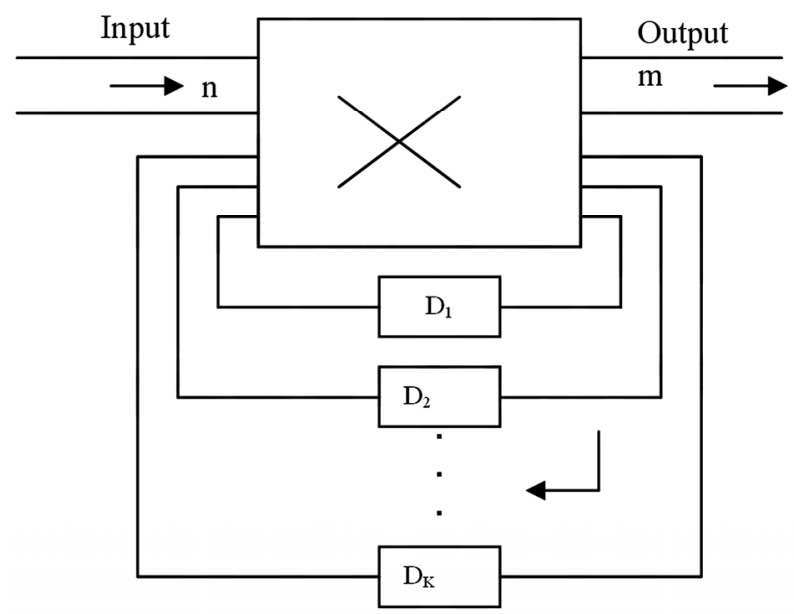

Figure 1. Recirculating delay line optical.

when the desired output port becomes available. This is possible because of the availability of electronic randomaccess memory (RAM). There is no equivalent optical RAM technology; therefore, the optical packet switches need to adopt different approaches for contention resolution. Meanwhile, WDM networks provide one new additional dimension namely wavelength, for contention resolution. There have been studies in literature for utilizing the three dimensions of contention-resolution schemes: wavelength, time, and space.

In this paper we explore the contention resolution, based on time and propose a new scheduling algorithm for prioritizing the packets within the node. The optical buffers basically delay the incoming signal by making it to travel a small distance, so as to provide some time to the processor for serving them in case the service is not available initially. Now this delay can be provided in fixed quanta's only. This unique feature of optical buffers (unlike their electronic counterpart which 'store' a packet) makes it necessary to have a minimum fixed delay once the packet has entered into the fiber delay line (FDL). Traditionally the buffer is implemented such that once the packet has entered into the FDL it suffers the delay and comes out after that time. The packet might be served had necessary arrangements been made or otherwise dropped. This architecture provides a single chance to server to serve it thus resulting in high packet loss. Ideally the packet should be available at all times at output after having entered the FDL (like equivalent electronic memory) so that it can be served whenever the resources are available.

Our new buffer architecture attempts to realize this objective by giving delays in steps of small granularity D ( $\mu \mathrm{Sec})$ which allows the packets to be processed if the resource at output is available otherwise reflected back to the FDL for multiple reflections as per the control algorithm.

It is already assumed that the number of output chan- nels (m) is less than the number of input channels (n) and therefore the queuing system has a fair chance of packet contention. The buffer works with a first-come firstserved (FCFS) scheduling policy and is implemented by means of FDL's with reflection.

In the proposed buffer architecture, when the packet arrives, it will be sent to the output node but if all output nodes are busy then it will be placed back in the first loop of the FDL having a delay of $\mathrm{D}_{1}$, after completion of the delay the packet competes for output port, failing this it will again be reflected back into the second delay of $\mathrm{D}_{2}$ and so on. The maximum delays that can be provided by using FDL's are assumed to have different values of delay such as a constant, arithmetic or a geometric progressive delay.

The flow chart for the packet servicing algorithm involving multiple delays in the proposed node architecture is presented in Figure 2. Obviously as a packet arrives at the node and the server is idle it is served immediately but these are queued if the server is busy. Usually the delays are kept finite by means of the FDL's, due to the limited time resolution related to the granularity and the new packet is going to be delayed at least by an amount of D for one loop circulation. Also the packet can't be made to reflect infinite number of times due to loss of energy at each reflection and hence is limited by accepted SNR.

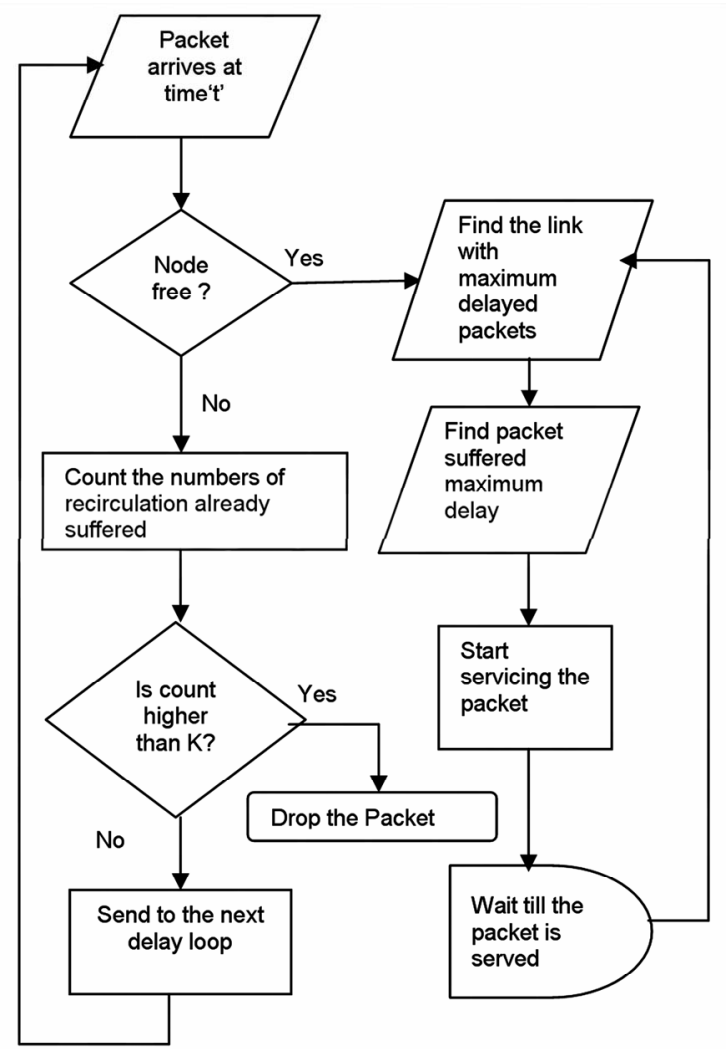

Figure 2. Flow chart for node performance analysis. 
Thus the packet is dropped after $\mathrm{K}$ reflections, which is modeled in terms of acceptable quality $\mathrm{q}$ and reflection $\operatorname{loss} \alpha$ as a function of $\log (q-\alpha)$. Considering the evolution of buffer contents over time, we can identify three important variables viz. order of bursts arrival, the packet inter arrival time (IAT) having Poisson distributed $\left(\mathrm{T}_{\mathrm{k}}\right)$ and the intermittent time between the $\mathrm{k}^{\text {th }}$ arrival and the next one.

This system is modeled for a random input, having an exponential service with $\mathrm{N}$ servers, an infinite number of prospective customers and a maximum queue length of $\mathrm{L}$. System probability for $\mathrm{j}^{\text {th }}$ call is expressed in term of packet arrival rate $\lambda$ and packet length $t_{m}$ as:

$$
\begin{gathered}
P_{j}(A)=P_{0}(A) \frac{A^{j}}{j !}, \text { for } 0 \leq j \leq N \\
P_{j}(A)=P_{0}(A) \frac{A^{j}}{N ! N^{j-N}} \text { for } N \leq j \leq N+L
\end{gathered}
$$

where $\mathrm{P}_{0}(\mathrm{~A})$ is used to make the sum of P's to unity assuming $A$ as $\lambda t_{m}$. Further $P_{0}(A)$ can be written as:

$$
P_{0}(A)=\left[\sum_{j=0}^{N} \frac{A^{j}}{j !}+\frac{A^{N}}{N !} \sum_{j=1}^{L} \frac{A^{j}}{N^{j}}\right]^{-1}
$$

In the proposed algorithm an incoming packet will be blocked if all the servers are busy \& queue is full. However the packet will be delayed if the servers are busy but queue is not completely full. The probability that $(\mathrm{N}+\mathrm{L})$ incoming packet is delayed can be written as

$$
P_{1}=\sum_{j=N}^{N+L-1} P_{j}(A)
$$

Further a packet will be serviced immediately if there are less than $\mathrm{N}$ packets in the system and the probability of immediate service of packet is expressed as

$$
P_{I S}=\sum_{j=0}^{N-1} P_{j}(A)
$$

The waiting time distribution for the incoming traffic can be expressed using the standard equation [14] as

$$
P=P_{N}(A) \sum_{j=0}^{L-1} \frac{\rho^{j}}{j !} \int_{N t / t_{m}}^{\alpha} x^{j} e^{-x} d x
$$

These equations have been used in throughput simulation in the MATLAB environment under the appropriate node and traffic assumptions.

\section{Simulation and Results}

Traffic throughput of the offered traffic that gets processed through the node has been estimated under various node design parameter constraints. This traffic has been evaluated using Equations (2-6) for the proposed node operated under traffic resolution algorithm. Figure 3 presents the carried traffic corresponding to incoming offered traffic with the variation of number of delay lines (N) involved. The simulated curve shows a linear dependence of the carried traffic on the offered traffic only upto a specific input load but beyond that it deteriorates owing to the rise in the blocking probability. Moreover increased incoming traffic results a crowded node forcing to reject the excess traffic. This qualitative behavior is also supported by the simulation curve showing a rejection beyond a critical offered traffic viz. for $\mathrm{N}=6$ beyond 2 Erlang.

The Figure 3 reveals better throughput is available if the delay is varied for different passes instead of keeping it constant for all passes. Basically if the delay is increased in every recirculation by a certain amount then it requires less number of recirculation comparing the fixed delay case to achieve a same particular amount of delay. As we have already discussed that recirculation of optical signal in the fiber delay line causes attenuation of signal power, insertion of different noises which ultimately affects the throughput of the network so it is better to have less number of recirculation to achieve better output. It may also be inferred from Figure 3 that the region of offered traffic for which the throughput is very high or the length of the high throughput region is greater in case of fixed delay network comparing to the variable delay system.

The Figure 4 depicts that, as the holding time increases the throughput decreases for all types of delay systems. Holding time corresponds to the processing speed and it increases for slower processing speed. Delay line will provide an amount of delay to the signals which are in the queue of getting served. Fast servicing will provide lesser processing time which in turn reduces the number

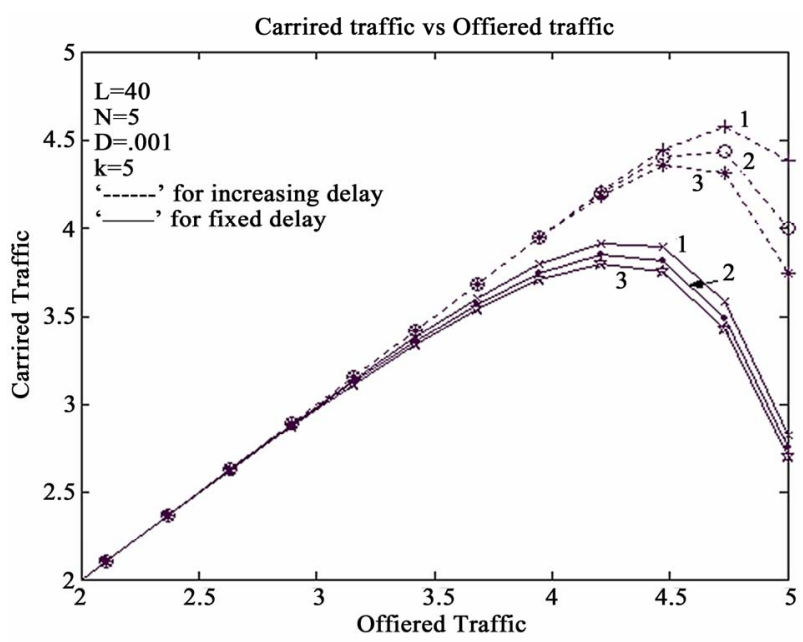

Figure 3. Plot of carried traffic vs offered traffic for different values of $N$. 
of recirculation in the delay loop. From Figure 4, it is also seen that the spreading of the linear region is greater in case of fixed delay loop comparing to the variable delay loop.

Figure 5 shows the variation of throughput for different numbers of recirculation loop. It is observed that as the number of recirculation loop increases the amount of carried traffic increases for both types of delay system i.e, for fixed delay as well as for increasing delay system. This is because the lesser loop increases the holding probability of the packet in the delay line. This in turn reduces the packet dropping probability. Throughput improvement is significant increasing in case of increasing delay system; this is obvious because the amount of delay achieved during recirculation increases gradually. It may be noted that the amount of region with maximum throughput is available in case of fixed delay system.

The analysis has been made more general by including a geometrical progressing delay loop in addition to arithmetic and constant delay lines. The corresponding throughputs have been presented in Figure 6 The fig reveals that the throughput improves as the delay increases which is expected but the increment of throughput will sustain up to a certain value of incoming traffic, after which the output decreases, means the packets which are coming further are being completely rejected.

From Figure 6 we can also infer that the insertion of more delay in the loop will increase the cost and complexity of the system as well and it is tolerable up to a certain limit. Thus this investigation will help the network designer to take a decision on the possible maximum throughput and the complexity of node architecture design.

\section{Conclusions}

The problem of wavelength contention in packet switched

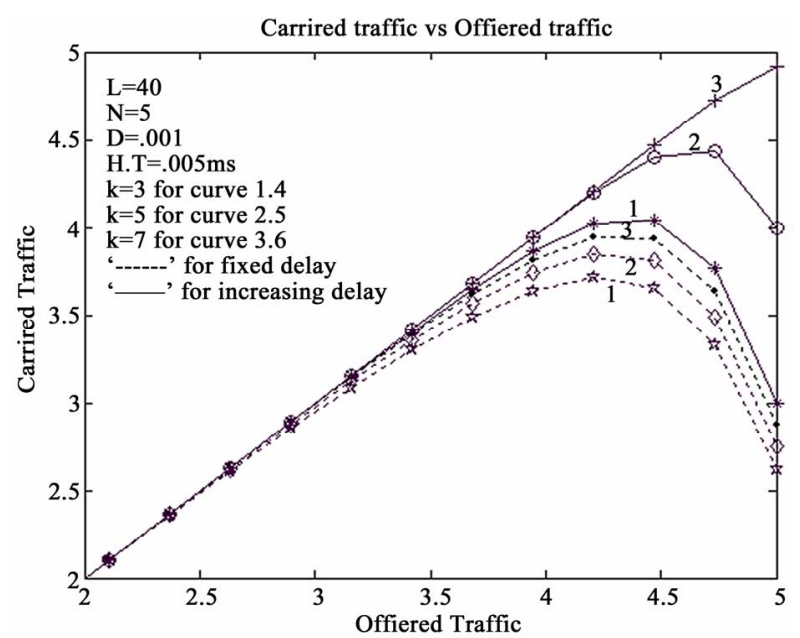

Figure4. Carried traffic vs offered traffic for different values of holding time.

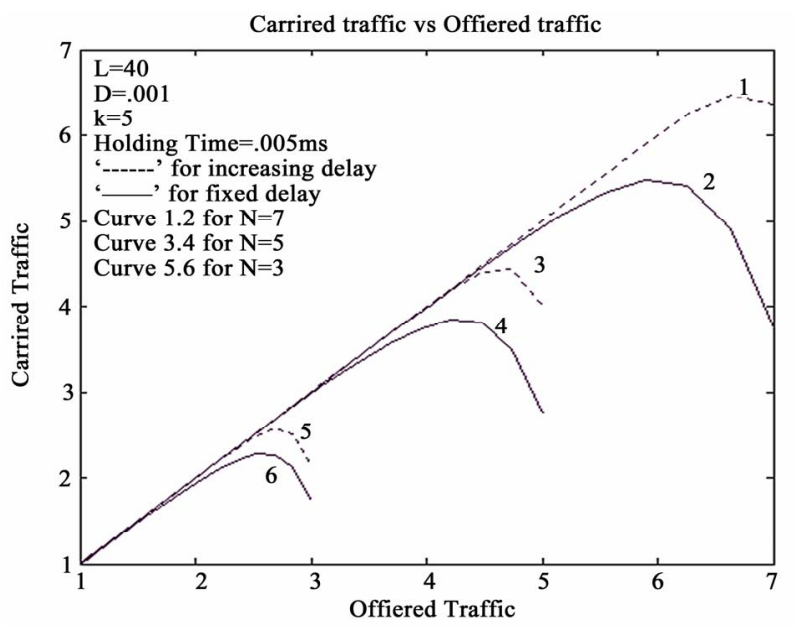

Figure 5. Plot of carried traffic vs offered traffic for different values of $k$.

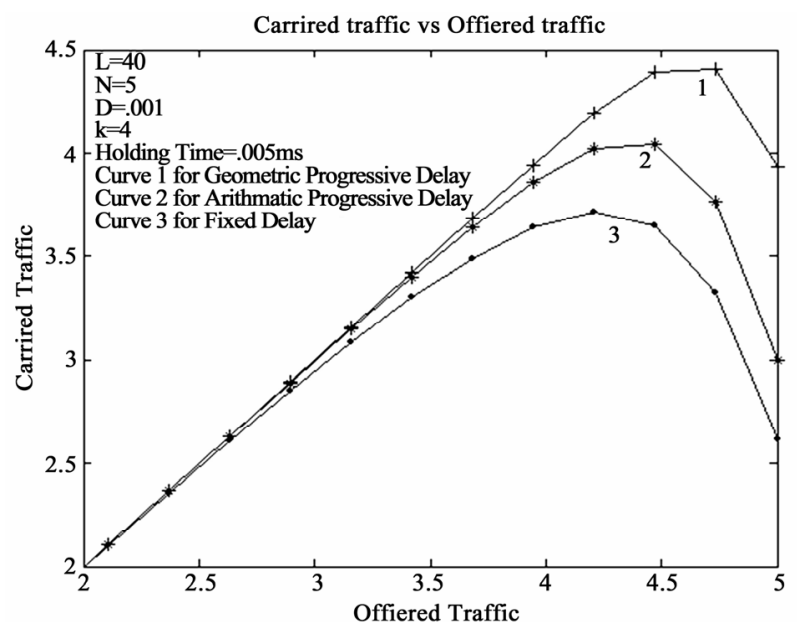

Figure 6. Plot of carried traffic vs offered traffic for different types of delay.

WDM networks using recirculation optical delay lines has been developed. The proposal is based on putting priority to the packets which have suffered maximum delay on the link in processing by using a proposed contention resolution algorithm. The performance of the algorithm has been evaluated using MATLAB simulation to establish a better contention resolution using a varied delay lines at the nodes. The analysis presented here is useful to predict the traffic throughput range of a processing node with given number of FDL's and relevant design parameters.

\section{References}

[1] L. Xu, H. G. Perros, and G. Rouskas, "Techniques for optical packet switching and optical burst switching," IEEE Comm. Magazine, pp. 136-142, 2001.

[2] S. L. Danielsen, et al., "Analysis of a WDM packet switch with improved performance under bursty traffic 
conditions due to tunable wavelength converters," J. Lightwave Technology, Vol. 16, No. 5, pp. 729-735, 1998.

[3] G. Shen, et al., "Performance study on a WDM packet switch with limited-range wavelength converters," IEEE Comm. Letters, Vol. 5, No. 10, pp. 432-434, 2001.

[4] Y. Yang, J. Wang, and C. Qiao, "Nonblocking WDM multicast switching networks," IEEE Trans. Parallel and Distributed Systems, Vol. 11, No. 12, pp. 1274-1287, Dec. 2000.

[5] D. K. Hunter and I. Andronovic, "Approaches to optical internet packet switching," IEEE Comm. Magazine, Vol. 38, No. 9, pp. 116-122, 2000.

[6] C. Develder, M. Pickavet, and P. Demeester, "Assessment of packet loss for an optical packet router with recirculating buffer," Proc. Conf. Optical Network Design and Modeling, pp. 247-261, 2002.

[7] W. Lin , R. S. Wolff and B. Mumey, "A markov-based reservation algorithm for wavelength assignment in alloptical netwrks," Journal of Lightwave Technology, Vol. 25, No. 7, pp. 1676-1683, July 2007.

[8] V. K. Chaubey and K. Divakar, "Modeling and simulation of WDM optical networks under traffic control protocols," Optical Fiber Technology (Elsevier), Vol. 15, pp. 95-99, Jan. 2009.

[9] J. Li , C. Qiao, J. Xu and D. Xu, "Maximizing throughput for optical burst switching networks," IEEE/ACM Transactions on Networking, Vol. 15, Issue 5, pp. 1163-1176, October 2007.

[10] R. Kundu, V. K. Chaubey, "Analysis of optical WDM network topologies with application of LRWC under symmetric erlang - C traffic," Novel Algorithms and Techniques in Telecommunications, Automation and Industrial Electronics, pp. 468-473, Aug. 2008.

[11] R. Ramamurthy and B. Mukherjee, "Fixed-alternate routing and wavelength conversion in wavelength-routed optical networks," IEEE/ACM Transactions on Networking, Vol. 10, No. 3, pp. 351-367, June 2002.

[12] Y. Zhou and G. S. Poo, "Multicast wavelength assignment for sparse wavelength conversion in WDM networks," INFOCOM'06, 25th IEEE International Conference on Computer Communications Proceedings, pp. 110, April 2006.

[13] P. RajlakshmiN and A. Jhunjhunwala, "Anlytical tool to achieve wavelength conversion performance in no wavelength conversion optical WDM networks," IEEE International Conference on Communications, ICC'07. pp. 2436-2441, 24-28 June 2007.

[14] A. O. Allen, "Probability, statistics, and queueing theory with computer science applications," Academic Press INC, USA, 1990. 